\title{
L'UlTima PARTE DEL CANZONIERE SABIANO
}

L'ultima parte del Canzoniere di Umberto Saba comprende nell'edizione definitiva ${ }^{1}$ le raccolte:

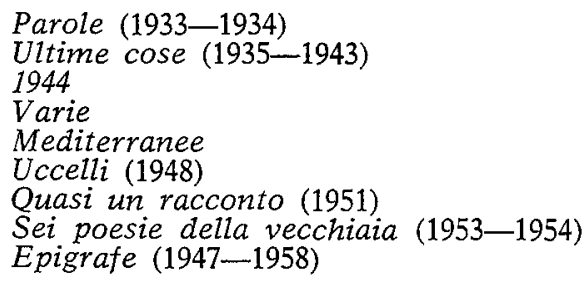

I primi quattro titoli figuravano come "Volume terzo« già nel tripartito Canzoniere del $1945^{2}$ che riportava - come dice la prefazione dell'editore »tutta l'opera poetica di Umberto Saba « ${ }^{3}$ fino allora pubblicata, Mediterranee, nate dopo, potevano essere inserite appena nel Canzoniere del $1948,{ }^{4}$ le raccolte che seguono poi furono aggiunte soltanto nell'edizione postuma del Canzoniere, sempre secondo l'intenzione dell'autore, come ci assicurano i curatori. ${ }^{5}$

Come possiamo desumere dalle stesse indicazioni cronologiche che accompagnano i titoli, ${ }^{6}$ le raccolte dell'ultima parte del Canzoniere sono frutto di un periodo relativamente lungo e dei piú difficili nella vita di Saba; per cui si spiegano le difficoltà che comporta la ricerca di denominatori comuni capaci di offrire una visione coerente dell'insieme.

In altre circostanze la creatività del Nostro si sarebbe probabilmente esplicata in un andamento meno turbato. Le atroci esperienze che gli riserbava l'età ormai prossima alla vecchiaia significarono un intervento esterno troppo duro persino per un poeta che una volta aveva pensato poter dire di se:

Lui le cose conquidono, ma poco, che sulla superficie della terra fanno col sangue gli uomini o per gioco. in fondo scava, in fondo è il suo tesoro; nel cuore della Terra, un cuore d'oro.?

1 U. Saba, Il Canzoniere (1900-1954), Torino, Einaudi, 1965.

2 U. Saba, Il Canzoniere (1900-1945), Roma, Einaudi, 1945.

${ }^{3}$ Ivi, p. 7.

4 U. Saba, Il Canzoniere (1900-1947), Torino, Einaudi, 1948.

${ }^{5}$ Nota alla presente edizione, II Canzoniere $(1900-1954)$, op. cit., p. V.

- Il 1944 figura per se stesso da titolo, come si vede.

7 "L'egoistau: Il Canzoniere (1900-1954), op. cit., p. 171. 
I fattori esterni non ebbero mai un ruolo cosí diretto ed evidente nella produzione sabiana come negli ultimi decenni. Il che si riflette nelle poesie del tempo in modi diversi. Il terzo volume è molto meno omogeneo del secondo: se per i componimenti degli anni venti non è difficile individuare un comune denominatore, per le raccolte che seguono il volume secondo, anche restando al Canzoniere del 1945, non riusciamo a stabilire subito un termine analogo capace di riassumere come un tutto le liriche del periodo in questione. Seguendo la produzione sabiana dagli anni trenta in poi, risulta sempre piú evidente la necessità di affrontare l'esame delle singole raccolte tenendo conto anche della situazione politica che ora quanto mai la condiziona nei contenuti e nelle forme.

Il passaggio dalla seconda alla terza parte del Canzoniere è persino piú singolare che non quello tra le prime due. La novità con cui è introdotto il terzo volume è rispetto a Il Piccolo Berto e Fughe ancor piú manifesta di quella di Canzonette nei confronti delle due raccolte precedenti. Le pagine ridotte a componimenti epigrammaticamente concisi che vi troviamo dopo l'ampiezza di scrittura della parte centrale del Canzoniere ci fanno rilevare già a prima vista una tensione lirica affatto diversa. Le novità sono individuabili nelle scelte lessicali, nella struttura della frase, nelle soluzioni ritmiche delle poesie con cui si apre l'ultimo volume del Canzoniere.

Negli anni trenta il poeta aveva imboccato una nuova strada. Alla poetica del procedimento riassuntivo che nella dialettica di Fughe aveva reggiunto l'apice delle potenzialità espressive subentra un nuovo tipo di scrittura. Che nome dare alla novità propostaci da Saba con Parole? II più rispondente sembrerebbe quello di "poetica della parola che è poi il termine con cui gli storici della letteratura riassumono le istanze di tutta la lirica italiana del tempo. Negli anni trenta anche Saba accetta di cimentarsi nelle possibilità espressive offerte da »ogni parola singola ${ }^{8}$ In che misura il triestino sia ligio all'assunto dell'avanguardia è testimoniato già nel titolo Parole. E come il titolo vada inteso lo possiamo desumere anche da questa sua osservazione: "Dalla prima poesia di Parole in poi, Saba, dà il suo pieno valore alla parola; da tale volontà e consapevolezza è nato non solo il libro, ma anche il titolo del libro«. ${ }^{9}$ Il senso del titolo è ulteriormente spiegato nel primo componimento, Parole, che fa da motto e prologo alla raccolta. L'apostrofe:

Parole,

dove il cuore dell'uomo si specchiava

- nudo e sorpreso - alle origini; ${ }^{10}$

rievoca i noti versi dell'ungarettiano Girovagoll e invocazioni simili tipiche di un tempo in cui la parola è fatta oggetto di interpretazioni affatto mistiche. Né il poeta si limita alla evocazione magica della parola; da ogni componimento risulta particolarmente evidente come »dà il suo pieno valore alla parola«.

${ }^{8}$ Dall'introduzione di G. Ungaretti a Sentimento del tempo, Milano, Mondadori, 1943, p. 20.

${ }_{9}$ U. Saba, Prose, Milano, Mondadori, 1964, p. 584.

${ }^{10}$ Il Canzoniere (1900-1954), p. 411.

${ }^{11}$ G. Ungaretti, L'Allegria, Milano, Mondadori, 1942, pp. 105-106. 
Eloquente quanto mai già la scelta delle parole, ben più rigorosa che per l'addietro. A leggere le poesie degli anni venti ci si imbatte spesso in

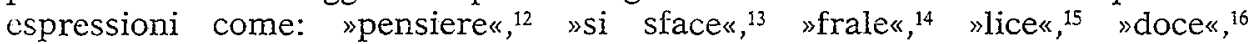
»chiome $\ll,{ }_{17}$ »fuore $\ll,{ }^{18}$ "novelli $\ll,{ }^{19}$ »aere ${ }^{20}$ Né sono infrequenti i troncamenti del tipo: »allettar «, ${ }^{21}$ »destin «, 22 »ciel«. ${ }^{23}$ Non potremmo dire che tali espressioni fossero tipiche di Saba o che fossero particolarmente felici. Il fatto è che il nostro non guardò mai al tradizionale armamentario poetico come ad esempio il Gozzano che se ne serviva soltanto a fini parodistici. Quando parla delle licenze poetiche e degli arcaismi che si era permesso, Saba cosí li legittima: »inseriti nel linguaggio piano e familiare, stuonerebbero molto sgradevolmente, se il calore dell'ispiranzione che investe da capo a fondo il vasto componimento, non ne attenuasse, fino a renderla appena percepibile, la sgradevolezza.$^{24}$ Le singole "mende«, a sua detta si perderebbero nell'insieme compositivo: "la passione, la tensione lirica che si esprime senza un momento di stanchezza, nei quindici sonetti, fa perdonare tali mende, li lava da macchie.$^{25}$ In altre parole insomma il poeta si richiama al tutto che è costituito dalla raccolta; ha rischiato avendo presente il tutto e pertanto lo si giudichi a pubblicazione avvenuta. L'osservazione appena citata si riferisce al ciclo di 15 sonetti che costituiscono Autobiografia. Il principio che Saba qui sostiene è applicabile solamente alle poesie degli anni venti quando la sua scrittura era incentrata non sull'elaborazione di frammenti ma di ampi e complessi cicli in cui le singole poesie erano in funzione di strofe. Parole presuppone procedimenti diversi. Intanto al primo posto è la parola; la raccolta quale insieme non risulta più struttura di tipo concluso bensí aggregazione di esternazioni liriche possibili in qualsiasi disposizione. Il curatore di antologie non ebbe mai mano cosí libera per quanto riguarda Saba come nella scelta delle poesie del nostro scritte nel decennio 1933-1943, vale a dire con le raccolte Parole e Ultime cose cui è comune il procedimento creativo. "Mende« tollerabili negli ampi cicli della parte centrale del Canzoniere, nel Saba di Parole e Ultime cose, ammonisce il poeta, "sarebbero state mortali, o quasi ". ${ }^{26}$ In Parole sarebbero dunque prevalsi criteri piú severi nella scelta e collocazione delle parole da parte del poeta.

Con Parole il discorso sabiano si fa essenziale. Qui non vi è piú spazio per perifrasi ed inicisi esplicativi; il periodo articolato è sostituito dall' enunciato conciso; le scene sono appena abbozzate, le figure mai disegnate a tutto tondo ma rappresentate con pochi tratti, rese con alcune linee. A

12 »Il vino«, Il Canzoniere (1900-1954), p. 219.

13 "L'incisore $\ll, I v i$, p. 229.

${ }^{14}$ "Chiaretta«, Ivi, p. 232.

15 »Autobiografia«, 1, Ivi, p. 243, e »II Silenzioso«, Ivi, p. 275.

16 "Autobeografia«, 5, Ivi, p. 247.

17 »Autobiografia«, 6, Ivi, p. 248.

18 »Autobiografia«, 7, Ivi, p. 249, e »Autobiografia«, 15, Ivi, p. 257.

is »Autobiografia«, 9, Ivi, p. 251.

20 "Il melanconico«, Ivi, p. 272.

21 "Fanciulle«, 1, Ivi, p. 279.

22 "Sopra un mio antico tema«, Ivi, p. 237.

${ }^{23}$ Dalla stessa poesia, Ivi, p. 238.

${ }^{24}$ U. Saba, Prose, op. cit., p. 505.

${ }^{25}$ Ivi, p. 506.

${ }^{26}$ Ibidem. 
illustrazione di questo procedimento che il nostro chiama »lavoro di sintesi «27 ci sia concesso citare Frutta erbaggi:

Erbe, frutta, colori della bella stagione. Poche ceste ove alla sete si rivelano dolci polpe crude.

Entra un fanciullo colle gambe nude, imperioso, fugge via.

\section{S'oscura}

l'umile botteguccia, invecchia come una madre.

Di fuori egli nel sole

si allontana, con l'ombra sua, leggero. ${ }^{28}$

Per il soggetto trattato la poesia permette innumerevoli confronti in quanto è uno dei piú vecchi e frequenti motivi della poesia di Saba. Anche nei particolari non è difficile individuare il modello che già con la raccolta Coi miei occhi aveva cominciato a ripetersi e che in Figure e Canti si proponeva come figura stereotipa. Identico anche l'ambiente mentre tanto piú evidenti sono le differenze nell'elaborazione. Saba che una volta indugiava sui particolari riportando dialoghi e pensieri che via via lo assalivano, parla ora un linguaggio che a malapena riusciamo a riconoscere. Lo stile del poeta si è fatto nominale; l'ambiente in cui dovrà svolgersi la scena è appena suggerito da due proposizioni ellittiche, la seconda delle quali legata »par plus d'un fil« alla strofa seguente; ogni parola vi è soppesata, sfruttata al massimo e collocata in una serie calcolata con estrema precisione. In una struttura cosí coerente anche le pause risultano ponderati mezzi stilistici: la seconda strofa si compone di tre brevi enunciati conclusivi e, perché la loro concisione trovi maggior rilievo, il poeta li scrive separatamente in forma di tre capoversi che si configurano quali insiemi conclusi.

La novità che dal punto di vista linguistico caratterizza Parole e Ultine cose è evidente da ogni proposizione, ogni singolo verso. Per citare qualche esempio. In versi come:

\section{s'incrociavano grida ch'eran razzi..$^{29}$}

cari nomi lanciavano i fanciulli, ad uno ad uno, come frecce. ${ }^{30}$

Mi fa un caffè come un trionfo, e i buoni occhi in volto gli ridono sportivi. ${ }^{31}$

\section{Baracconi}

non hanno mani a vendere la sera le indigeste castagne ai ragazzoni della libera uscita. ${ }^{32}$

non è difficile riconoscere ciò che parlando della genesi delle raccolte Parole e Ultime cose il poeta chiama »lavoro di sintesi«. E non è forse un tipico modulo di sintetizzazione già il traslato che vi si ripete?

${ }^{27} I v i$, p. 607 .

${ }^{28}$ Il Canzoniere (1900-1954), p. 437.

29 "Tre momenti«, Il Canzoniere (1900-1954), p. 421.

so „Fanciulli allo stadio«, Ivi, p. 423.

${ }^{31}$ „Sobborgo«, Ivi, p. 435.

32 »iazza«, Ivi, 463. 
L'analogismo che salta agli occhi nei versi sopra citati è il segno che legittimamente richiama gli esempi che il triestino trova negli ermetici. Peraltro le frasi citate mostrano altresí quanto Saba abbia approfittato dei modelli della poesia italiana degli anni venti e trenta senza per questo rinnegare se stesso. I primi due esempi sono presi da poesie che esprimono il caratteristico "compalpitare « ${ }^{33}$ con la folla: in altre parole si tratta di un motivo che conosciamo già dagli esordi sabiani e specie dalle raccolte Coi miei occhi e La serena disperazione. Goal:

Il motivo, antichissimo, trova il massimo risalto nella poesia intitolata

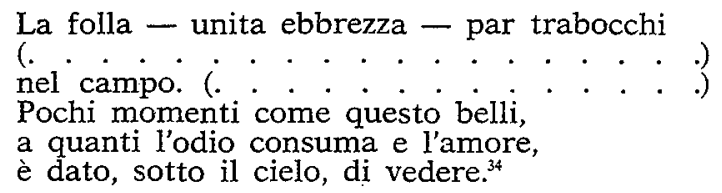

Il sentimento che ha la sua definizione più precisa nell'espressione »unita ebbrezza" risalta anche alla conclusione della poesia Tre momenti:

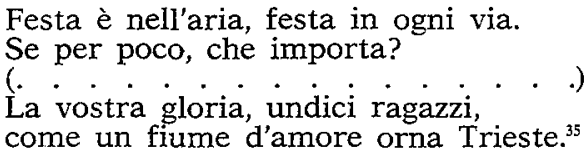

In motivi come quello dei "ragazzi« d'altro canto non abbiamo difficoltà a riconoscere $\mathrm{i}$ simboli di una primordiale gioia di vivere, motivo che nella poetica sabiana si esplicita assieme all'aspirazione "d'immettere la sua dentro la vita / di tutti«. ${ }^{36} \mathrm{E}$ versi come "La sua gioia si fa una capriola « ${ }^{37}$ sono certamente quelli che maglio illustrano la novità di Parole. Al lettore poi che conosce l'intera opera sabiana queste proposizioni richiamano anzi tutto poesie come Scherzo $0^{38}$ o In cortile, ${ }^{39}$ benchè la differenza tra $\mathrm{i}$ sonetti del 1908 e i componimenti degli anni trenta sia a prima vista tale da sconsigliare ricerche di denominatori comuni.

Nella matrice delle costanti peculiari della tematica del nostro rientra, a una considerazione attenta, anche il prologo della nuova raccolta. Nella poesia Parole che a prima vista suona come invocazione di un ermetico, Saba rimane fedele alla vecchia concezione della parola. Le »origini« cui anela sono altra cosa che l'ungarettiana "vita iniziale ${ }^{40} \mathrm{E} \mathrm{l}$ ' "oasi che il triestino cerca non è certamente il "paese innocente ${ }^{41}$ sognato da Ungaretti ed epigoni. La catarsi che Saba si ripromette quando dice:

${ }^{33}$ L'espressione è usata da Saba. V. Prose, p. 591.

${ }^{34}$ Il Canzoniere (1900-1954), p. 424.

${ }^{35}$ Ivi, p. 421.

${ }^{36}$ "Il borgo«, Ivi, p. 314.

37 „Goal«, Ivi, p. 424.

${ }^{38}$ Il Canzoniere (1900-1954), p. 56.

${ }^{39}$ Ivi, p. 48.

${ }^{40} \mathrm{G}$. Ungaretti, Girovago, op. cit., p. 424.

${ }^{41}$ Ibidem. 
(. . . . . . . . . . . .) Insieme

delle memorie spaventose il cumulo

si scioglierebbe, come neve al sole.2

è quella indotta da una "chiarificazione interna ${ }^{43} \mathrm{La}$ condizione di un "illimpidimento della forma «44 è per lui, cantore di Parole, willimpidimento psicologico ${ }^{45}$ Onde gli esiti cui giunge Saba persino quando il suo »modus operandi« è peraltro più aderente al gusto del tempo: a differenza dei contemproranei italiani ed europei cui l'indeterminatezza è assurta, a detta di Hugo Friedrich, a principio estetico, ${ }^{46}$ il nostro non rinnega mai il postulato della chiarezza. "Noi«, dice Saba, "non amiamo (...) l'ermetismo perché sappiamo che esso nasconde un processo (psicologico) involutivo anziché evolutivo, e il mondo ha piú bisogno di chiarezza che di oscurità巛. ${ }^{47}$ In questa precisazione non è difficile individuare le prese di posizione esposte già nel saggio "Quello che resta da fare ai poeti «48 del 1911, nuovi sono soltanto i presupposti su cui fa leva il triestino quando postula la chiarezza. La percezione che »il non poter vedere chiaro in noi stessi" rappresenta "l'equivoco della nevrosi, [...] due buoni terzi dei mali che affliggono l'uomo « è frutto dell'interesse per la psicoanalisi da cui, come dice lo stesso Saba, ne verrà "molto più illuminato su se stesso e gli altri«. ${ }^{50} \mathrm{La}$ "grande chiarificazione interna ${ }^{51}$ gli giunge proprio negli anni trenta per riflettersi non soltanto nelle poesie ma anche nei contemporanei aforismi ${ }^{52}$ che meglio di altre sue cose illustrano come siano da interpretare le promesse date nel già citato prologo quando prefigura che:

delle memorie spaventose il cumulo si scioglierebbe, come neve al sole.

Una "scorciatoia« si chiude con queste parole: »Ma se tu, se io, potessimo portare quegli inconsci conflitti alla luce della coscienza, ne proveremmo un grande, un indicibile sollievo; e quelli si risolverebbero - scoppierebbero - in aria, come bolle di sapone.$^{53}$ Osservazione che è per cosí dire la replica letterale di quanto Saba ha detto nei versi del prologo di Parole e che mostra con sufficiente chiarezza qual è il suo rapporto nei confronti dei poeti che vedono nel mistero il fascino maggiore della poesia.

Eppure Parole e Ultime cose sono da un certo punto di vista anche contenutisticamente in consonanza col tempo in cui furono scritte. Non condividiamo quindi la tesi di quegli storici della letteratura che la novità delle due raccolte la ascrivono solamente alla necessità del poeta di aggior-

42 "Parole«, Il Canzoniere (1900-1954), 411.

43 U. Saba, Prose, op. cit., p. 580.

${ }^{44}$ Ibidem.

${ }^{45}$ Ivi, p. 581 . p. 178

${ }^{46}$ Hugo Friedrich, Die Struktur der modernen Lyrik, München 1968, Rowohlt,

${ }^{47}$ Prose, pp. 640-641.

48 Ivi, pp. $751-759$.

${ }^{49}$ Ivi, 788 .

${ }^{50} I v i$, p. 786.

5 Ivi, p. 580 .

52 Alludiamo a Scorciatoie che Saba incomincia a scrivere appunto nel 1934. Si vedano Primissime scorciatoie, 1934-1935 (Prose, pp. 367-385) e Scorciatoie rifiutate, 1934-1935 (Prose, pp. 387-391).

${ }_{53}$ Prose, p. 309. 
narsi formalmente, e che si limitano alla considerazione degli »stilemi« che Saba mutuerà dalla "poetica del suo tempo". Se lo stato psichico in cui Saba venne a trovarsi negli anni trenta non avesse avuto notevoli analogie con la coscienza da cui nacque la poesia ermetica, non avrebbero potuto prender corpo Parole e Ultime cose. Il termine piú idoneo a definire tale stato noi lo vediamo nel titolo che il poeta si proponeva di dare alla raccolta delle poesie scritte tra il 1933 e il 1934. Raccolta che in un primo tempo Saba aveva inteso chiamare "Distacco" e che, come dice Iui stesso, ${ }^{54}$ solo successivamente decise di intitolare »Parole«. Con questo secondo titolo passa in primo piano l'aspetto formale della raccolta: l'esperienza da cui nascono le poesie che la compongono aveva la designazione migliore nel titolo originario. E quando parliamo delle analogie esistenti fra il triestino e i poeti del suo tempo il titolo originario non è per niente meno indicato a stabilire tali affinità di quello che alla fine prevalse. Distacco è un titolo di cui si serví anche Ungaretti quando scrisse:

\section{ECCOVI UN'ANIMA \\ DESERTA \\ UNO SPECCHIO IMPASSIBILE ${ }^{55}$}

termini che avrebbe potuto applicare a se stesso anche il Saba del periodo che inizia con Parole.

Che »distacco« sia il motto piú adatto a designare l'atteggiamento nei confronti della realtà che Saba ha negli anni trenta lo indicano già i primi componimenti di Parole. Basti pensare a effusioni come questa:

Neve che cadi dall'alto e noi copri, coprici ancora, all'infinito. Imbianca la città con le case e con le chiese, il porto con le navi; le distese

dei prati, i mari agghiaccia; della terra

fa - tu agusta e pudica - un astro spento, una gran pace di morte. ${ }^{56}$

oppure

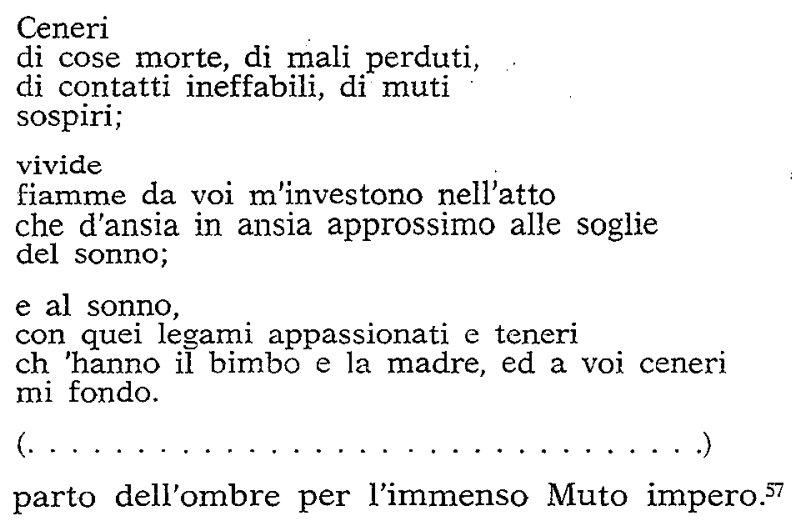


Su una posizione di osservatore distaccato il poeta viene a trovarsi persino quando gli si offre "un'ultima possibilità (...) di ,compalpitare con gli altri« ${ }^{58}$ Una delle poesie scritte »per il gioco del calcio« cosí chiude:

\author{
Piaceva \\ essere cosí pochi intirizziti \\ uniti, \\ come gli ultimi uomini su un monte, \\ e guardare di lá l'ultima gara. ${ }^{59}$
}

Se sono ancor sempre individuabili i vecchi motivi, ne esce visibilmente mutata la funzione:

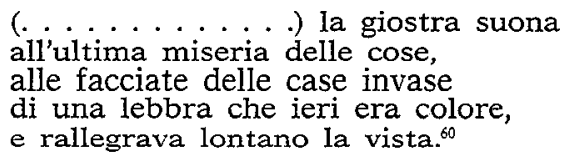

Le »cose di un tempo assumono, come dice Saba a chiusura di una poesia, una diversa connotazione:

$$
\text { ( altra costellazione - un'altra età. }{ }^{61}
$$

Ma piú che in Parole la nuova "Stimmung« trova espressione nella raccolta Ultime cose. In Parole l'maltra età " si esplicita in enunciati che già si contraddicono. Immagini come »astro spento«, "neve", "ceneri« in Parole costituiscono soltanto uno dei poli fra i quali oscilla lo stato d'animo del poeta. Persino in una sola poesia, a immagini "della fine del mondo «2 può succedere un "risveglio ${ }^{63}$ in cui si fanno sentire "acute / dilaceranti nostalgie «64 che hanno l'effetto di "quel rosso « ${ }^{65}$ della notissima Via della Pietà e moduli analoghi delle prime due parti del Canzoniere. Se in Parole autodefinizioni del tipo "Ulisse del declino ${ }^{66}$ si intrecciano al pensiero che

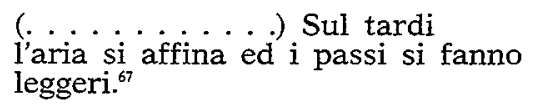

e l'»evasione « ${ }^{68}$ vi è sempre possibile, Ultime cose parlano soltanto di un tormento per il quale Saba non vede piú via d'uscita.

Nelle poesie che succedono a Parole

s8 Prose, p. 591.

59 "Tredicesima partita", Il Canzoniere (1900-1954), p. 422.

60 „Sobborgo«, Ivi, p. 435.

61 »Firenze«, Ivi, p. 433.

62 »Inverno«, $I v i$, p. 426.

${ }_{63}$ »Neve«, Ivi, p. 413.

${ }^{64}$ Ibidem.

os Il Canzoniere (1900-1954), p. 91.

* "Ulisse", Ivi, p. 419.

67 „Felicità«, Ivi, p. 430.

${ }_{68}$ Ibidem.

69 »Spettacolo«, Ivi, p. 476. 
(. . .) Si spogliano le cose,

se ne tocca lo scheletro. ${ }^{69}$

Qui non c'è piú spazio per le illusioni. La vista delle »belle cose come prede esposte ${ }^{70}$ lascia nel poeta nient'altro ormai che un "rimpianto oscuro ${ }^{71}$ Uno spazio sempre maggiore comincia ad assumere

La memoria

amica come l'edera alle tombe. ${ }^{72}$

il graduale deperimento è espresso con immagini che esclucono qualsiasi speranza di »risvegliok.

Ed è il pensiero

della morte che, in fine, aiuta a vivere. ${ }^{73}$

Infine viene a mancare anche quell'incondizionata volontà di vivere con cui Saba aveva combattuto la disperazione per passare dalle visioni piú angosciate all'esaltazione della gioia di vivere con quelle imprevedibili giravolte cosí caratteristiche per lui. Senza senso è detto qualsiasi tentativo di opporsi »allo stratempo $:^{74}$

Ti pare il sopravvivere un rifiuto d'obbedienza alle cose. ${ }^{75}$

Enunciati simili riscontriamo anche nei contemporanei di Saba: il distacco dalla realtà è avvertibile negli anni trenta in tutti i piú importanti poeti italiani. Il rifiuto della realtà è a detta degli storici della letteratura uno dei motivi centrali della poesia ermetica. "Rifiuto che si manifesta in vario modo. Espressione ricorrente è ad esempio l'»ansia precoce di morire ${ }^{76}$ o lo sfogo del poeta che si dice ${ }^{2}$ sradicato dai vivi«. ${ }^{77}$ Il motivo dell'estraniamento d'altro canto non si presenta in nessun poeta ermetico gravido delle implicazioni che ha in Saba.

Ultime cose parlano dell'angoscia di un uomo che la legge condanna alla condizione di "relegato fra i paria della società ${ }^{78}$ e quindi alla morte civile.

$\mathrm{E}$ ci sia concesso citare la poesia $D a$ quando interessante dalla visuale della nostra ricerca non fosse che per le immagini con cui opera il poeta:

Da quando la mia bocca è quasi muta

amo le vite che quasi non parlano.

Un albero; ed appena - sosta dove

io sosto, la mia via riprende lieto -

il docile animale che mi segue.

Al giogo che gli è imposto si rassegna.

Una supplice occhiata, al piú, mi manda.

Eterne verità, tacendo, insegna. ${ }^{79}$

70 Ecco, adesso tu sai«, Ivi, p. 452.

${ }^{71}$ Ibidem.

"Ultimi versi a Lina«, Ivi, p. 474.

73 "Sera di febbraio", Ivi, p. 471.

${ }^{74}$ »Porto«, Ivi, p. 484.

${ }^{75}$ "Il vetro rotto,$I v i$, p. 473.

${ }^{76} \mathrm{Si}$ veda per esempio la lirica "Vento a Tindari« della raccolta Acque e terre di Quasimodo (S. Quasimodo, Tutte le poesie, Milano, Mondadori, 1961, p. 29).

$7 \mathrm{~S}$. Quasimodo, "Al tuo lume naufrago" (Tutte le poesie, op. cit., p. 106).

${ }^{78}$ U. Saba, Prose, op. cit., p. 602.

${ }^{79}$ Il Canzoniere $(1900-1954)$, p. 461. 
Confessione da cui non è difficile intravedere che risale all'entrata in vigore dei cosiddetti "provvedimenti razziali« che per lo stigma di ebreo costringeva il poeta al silenzio. ${ }^{80}$ Dalle rimanenti poesie di Ultime cose pubblicate integralmente solo dopo la liberazione, non è altrettanto chiaro a cosa ascrivere il »congedarsi dall'arte e dalla vita ${ }^{81}$ da parte del poeta. Considerando però da vicino la cronaca delle circostanze in cui vennero scritte Ultime cose ci convinciamo non essere senza legame con quanto comportarono i famigerati provvedimenti anche i componimenti a prima vista cosí apolitici come Teatro. ${ }^{82}$

Le prime manifestazioni dell'angoscia che doveva successivamente crescere fino a farsi »incubo (...) particolarmenete spaventoso « ${ }^{83}$ lo si può dedurre già da Parole. Il caratteristico distacco di cui parlano Ultime cose è suggerito già in Parole che non senza motivo il poeta aveva voluto intitolare appunto "Distacco". Per indicare la »stimmung" all'insegna della quale inizia il terzo volume del Canzoniere difficilmente troveremmo espressione piú adatta del titolo che Saba intendeva dare originariamente alle poesie scritte fra il 1933 e il 1934. Nell'interpretare l'atteggiamento di Saba nel decennio in cui compose Parole e Ultime cose andranno tenuti presenti gli avvenimenti che in quel periodo sconvolsero l'Europa. Periodo a proposito del quale Moravia ebbe a dire: $\gg I$ dieci anni tra il 1933, anno dall'ascesa al potere di Hitler, e il 1943, anno della caduta del fascismo, furono dal punto di vista della vita pubblica, in peggiori della mia vita, e non posso ricordarmene ancora oggi senza orrore ${ }^{84}$ Anche per Saba, e per gli stessi motivi, col 1933 inizia il periodo delle esperienze piú cruciali. Le due raccolte del decennio 1933-1943 testimoniano in modo eloquente di come il triestino vivesse il tempo di cui parla Moravia. Sintomatiche specie Ultime cose in cui la parola chiave è »orrore«: "Il tempo gli orrori suoi precipita " 85 »dagli orrori / mi rifugio del giorno ${ }^{86}$

Gli ebrei triestini furono i primi ad avvertire il pericolo che incombeva sull'Italia e impegnarono tutto il proprio influsso per ostacolare l'avvicinamento tra Germania e Italia. ${ }^{87}$ Quando poi il fascismo lanciò la politica

so $\gg$ Come si sa, leggi razziali vietavano di pubblicare scritti di ebrei o ritenuti ebrei, sia in giornale $\mathrm{e}$ in rivista che in volume L'editore Mondadori si era invano rivolto a Roma per ottenere il permesso di pubblicare l'opera poetica del nostro. Alcuni periodici magari riuscivano ancora di tanto in tanto a presentare qualche poesia dell'autore proibito, ma una rivista studentesca, ad esempio, aveva tentato invano di farlo: »non è compito dei G. U.F. stampare gli scritti degli ebrei«, fu il rimprovero prevedibile che non tardò a giungere. Si veda S. Bon Gherardi, La persecuzione antiebraica a Trieste (1938-1945), Udine, Del Bianco, 1972, pp. 145-146.

${ }^{81}$ U. Saba, Prose, op. cit., p. 611.

$\therefore 2$ U. Saba, Il Canzoniere (1900-1954), op. cit., p. 465.

${ }^{83}$ U. Saba, Prose, op. cit., 608.

84 Autobiografia in breve di Alberto Moravia, cit. da O. del Bruno, Moravia, Milano, Feltrinelli, 1962, p. 13.

${ }^{85}$ U. Saba, Il Canzoniere (1900-1954), op. cit., p. 450 (»Amico«).

Ivi, p. 470.

87 Si veda la prefazione di Enzo Collotti al già citato libro di S. Bon Gerardi, La persecuzione antiebraica a Trieste, pp. 10-11. In una relazione che risale alla prima metà del 1938 il questore di Trieste faceva presente al Ministero degli Interni che "L'elemento ebraico a Trieste, numeroso e benestante, merita speciale considerazione non soltanto nei riflessi della vita economica cittadina (poiché come è noto quasi tutte le iniziative ed i posti direttivi della finanza e del commercio sono in sue mani) ma per l'atmosfera politica che direttamente crea e comunica 
razzista, Trieste »città ebraica per eccellenza«, 8 diventò l'epicentro dell'antisemitismo in Italia. Il direttore dell'»ebreo" Il Piccolo ${ }^{89}$ tentò invano di ricordare a Farinacci e agli altri corifei della campagna antisemita il ruolo avuto dagli ebrei triestini nel movimento irredentistico in cui »Felice Venezian dirigeva con diritta coscienza d'Italiano la politica adriatica, 90 invano gli faceva presenti le benemerenze di personaggi come Giacomo Venezian "fra i più puri fondatori del nazionalismo italiano ". ${ }^{11} \mathrm{Gli}$ ebrei, bollati quali »ebreo-democraticomassoni« a Trieste venivano relegati nella stessa categoria dei "rosso-slavi« che essi ebrei da borghesi quali erano per buona parte avevano aiutato a opprimere sotto il fascismo.92 Invano "Il Piccolo" allegava le benemerenze riconosciute dal Duce stesso.93 Farinacci con cui il giornale di Mayer disperatamente polemizzò produceva numeri insistendo sul fattore proporzioni: »Facendo le dovute proporzioni, fra i 250 mila cattolici e i 4 mila obrei, si deve concludere che questi hanno i nove decimi (900 su mille!) dei posti in cui si esprime la direzione intellettuale, economica, finanziaria e sindacale di Trieste ${ }^{94}$ A Trieste, il centro ebraico più influente del Paese, 25 s'erano fatti sentire accenti antisemiti fin dal 1933. $\mathrm{E}$ già a metà del 1934 in una lettera al federale di Trieste, Starace faceva rilevare le "congreghe ebraiche levantine che hanno in mano l'economia $e$

ad un esteso strato della popolazione (...). Tale clima pervade la città e di esso si può avere la sensazione attraverso l'aperta ostilità verso la nazione Germanica, persecutrice delle popolazioni di razza ebraica e la diffusa riluttanza quindi nel seguire le attuali direttive politiche di amicizia con la predetta nazione«. Cit. da S. Bon Gerardi, La persecuzione antiebraica a Trieste, op. cit., pp. $48-49$.

${ }^{88} \mathrm{R}$. De Felice, Storia degli ebrei italiani sotto il fascismo, Torino, Einaudi, 1962, pp. 14-15.

${ }_{s 9}$ Il giomale triestino $I l$ Piccolo veniva definito in una lettera a Mussolini come suno strumento dell'ebraismo nazionale e internazionale« ( $R$. De Felice, Storia degli ebrei italiani sotto il fascismo, op. cit., p. 304, nota n. 1.

${ }^{90}$ R. Alessi, "Situazioni che non pesano", Il Piccolo, 25. 1. 1938.

:I Ibidem.

92 "Nulla sarebbe meno esatto che voler vedere una estraneità dell'ebraismo al fascismo o volere mitizzare un antifascismo ebraico sin dalle origini della dominazione fascista. Nulla di tutto ciò, tanto meno a Trieste. La borghesia ebraica si adegua facilmente al piú generale comportamento della borghesia triestina, che non è di semplice e conformistica acquiescenza ma spesso di attiva adesione e di sostegno al nuovo regime, in nome di una solidarietà di classe che non sembra trovare limiti, al punto che si può senz'altro affermare che nel primo dopoguerra l'alta finanza ebraica cerca sicurezza nel fascismo e con il fascismo convivrà tranquillamente, condividendone anche la politica antislava. Anche qui la presenza tra gli antifascisti locali di ebrei come Bruno Pincherle (...) non è che l'eccezione che conferma la regola«, scrive Enzo Collotti nella già citata prefazione al volume La persecuzione antiebraica a Trieste di S. Bon Gerardi, pp. 8-9. Del resto, quando la campagna antisemita assunse carattere ufficiale, a Trieste anche il podestà dovette dimettersi perché ebreo. Ma nomi ebraici figurano fra gli stessi fondatori del fascio triestino e delle prime ssquadre d'azione «: basti pensare alla figura di Piero Jacchia. Vedi Tiberio, Il fascismo a Trieste negli anni 1919-1923). Udine, Del Bianco, 1956, p. 14 e p. 19; C. Silvestri, Storia del fascio di Trieste dalla origini alla conquista del potere (1919-1922), in Fascismo - guerra - resistenza. Trieste, Libreria Italo Svevo, 1969; M. Kacin Wohinz, Primorski Slovenci pod italijansko zasedbo, 1918-1921, Maribor, Založba Obzorja, 1972, p. 145 ed altri saggi sull'argomento.

${ }^{93} \mathrm{~V}$. il già citato articolo di Alessi, pubblicato su il Piccolo del 25 gennaio 1938.

${ }^{97}$ Dall'articolo "Seconda replica«, pubblicato da Farinacci su Il regime fascista. Renzo De Felice, Storia degli ebrei italiani sotto il fuscismo, op. cit., p. 306.

"s Trieste fu uno dei centri europei piú importanti del sionismo fin dagli inizi di questo movimento, e qui si pubblicava il foglio principale degli ebrei italiani. 


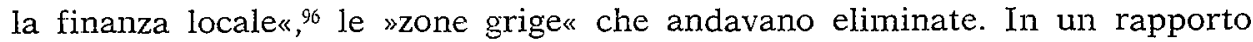
del 1937, Trieste è indicata quale sibrida zona dell'ebraismo massonico in camicia nera.$^{98}$ Con la promulgazione delle famigerate leggi sulla »difesa della razza« in nessun'altra città italiana quanto a Trieste gli ebrei furono esposti a tanta violenza, culminata, come noto, in veri e propri pogrom. ${ }^{99}$

Per cui fin dai primi del 1939 gli ebrei cominciarono a emigrare in massa. In un primo tempo pensò di emigrare anche il nostro che nella primavera del 1939 soggiornò per breve tempo a Parigi; pur tuttavia lo stesso anno tornò in patria rassegnandosi alla sorte che aveva colpito la comunità cui apparteneva in linea materna. Malgrado le possibilità di sottrarsi alle angherie rinnegando formalmente l'origine ebrea, Saba sopportò con dignità tutte le umiliazioni. 100 Nella città natia rimase fino all'autunno 1943 quando si vide costretto a fuggire e "nascondersi come una povera bestia inseguita a morte.$^{101}$

La liberazione trova Saba nell'agosto del '44 a Firenze, città che gli aveva offerto $»$ rifugio allo stratempo « ${ }^{102} \mathrm{Di}$ quest'esperineza testimoniano le poesie immediatamente successive a Ultime cose e che hanno il comune titolo di 1944:

Da una burrasca ignobile approdato

a questa casa ospitale, m'affaccio

- liberamente alfine - alla finestra.

Guardo $(\ldots \ldots \ldots)^{103}$

cosí inizia il primo dei cinque componimenti scritti quando il nostro »può, finalmente, affacciarsi alla finestra, senza il timore di essere riconosciuto da qualche zelante "patriota«, il quale in un accesso di amor patrio, e contro compenso di 5000 lire a testa, consegnasse lui e la sua famiglia ai tedeschi e alle camere a gas « ${ }^{104} \mathrm{E}$ Saba si lascia andare allo sfogo immediato. Alle allusioni del discorso ermetico subentra la parola franca che chiama le cose nella loro spaziale e temporale concretezza. La realtà politica non è più soltanto suggerita: Saba la enuncia explicitis verbis sia quando lamenta:

Tutto mi portò via il fascista abbietto

ed il tedesco lurco ${ }^{105}$

${ }^{96}$ Vedi R. De Felice, Storia degli ebrei italiani sotto il fascismo, op. cit., p. 172.

${ }^{79}$ Ibidem.

98 Ivi, p. 305.

${ }^{99}$ Manifestazioni di ostilità nei confronti degli ebrei si ebbero a Trieste fin dall'inizio del 1938; e da allora in poi le scene di violenza andavano assumendo aspetti sempre piú gravi. Anche l'atteggiamento antisemita delle organizzazioni si faceva sentire qui piú che altrove in Italia; particolarmente attiva si dimostrò la G. U. F., la cui rivista fu elogiata dallo stesso Preziosi come "grido di liberazione contro l'ebreo, il mezzo-ebreo, l'ebreo mimetizzato e contro i fascistoni agganciati agli ebrei« (Vedi S. Bon Gherardi, La persecuzione antiebraica a Trieste, op. cit., p. 143).

${ }^{100}$ Nato da un matrimonio »misto«, Saba avrebbe potuto trovar riparo contro le leggi razziali: gli sarebbe bastato un certificato di battesimo ricevuto prima dell'entrata in vigore delle leggi razziali; ma il senso dell'onore non permise al nostro di ricorrere a stratagemmi del genere.

${ }^{101}$ U. Saba, Prose, op. cit., p. 613.

${ }^{102}$ U. Saba, Il Canzoniere (1900-1954), op. cit., p. 484 ("Porto«).

103 Ivi, p. 489 (»Avevo«).

${ }^{104}$ U. Saba, Prose, op. cit., p. 615.

${ }^{105}$ U. Saba, Il Canzoniere (1900-1954), op. cit., p. 489 (»Avevo«). 
che quando con l'immediatezza fattografica dello storiografo annota:

Questo è il Teatro degli Artigianelli, quale lo vide il poeta nel mille

novecento quarantaquattro, un giorno

di settembre, che a tratti

rombava ancora il cannone, e Firenze

taceva, assorta nelle sue rovine..$^{100}$

La poesia-accusa Avevo e l'altra che inizia con la frase:

Falce e martello e stella d'Italia ornano nuovi la sala. $(. . .)^{107}$

mostrano in una luce nuova un Saba che finora aveva accuratamente evitato qualsiasi impegno politico. Con la raccolta 1944 il triestino si presenta nelle vesti di bardo della Resistenza. Il poeta non se ne sta più al di sopra degli avvenimenti che sconvolgono il mondo ma li tratta dalla visuale di una precisa partecipazione. Cosí all'uomo che finora s'era presentato sempre nella specia di homo natura si sostituiscono protagonisti storicamente definiti visti in una situazione politica che in quanto tale assorbe l'interesse del poeta. Qua e là si ha l'impressione che nella tematica del nostro, che ha finora tenacemente insistito con esclusività programmatica nella raffigurazione dell'uomo naturale, ormai prevalga del tutto l'aspetto storico.

Il peculiare storicismo cui Saba improvvisamente aderisce è evidente al confronto di due poesie improntate allo stesso motivo: mi riferisco ai Due madrigali per la Dichessa d'Aosta della raccolta Varie. ${ }^{108}$ Il primo, scritto a Trieste nel 1934, dice:

Cosí giovarie sei, cosí leggera

cammini incontro alla dubbia fortuna,

che se non fossi una

principessa,saresti una ragazza. ${ }^{109}$

Il secondo, composto a Firenze nel 1944, cosí inizia:

Penso le mani, le tue belle mani.

Sono passati per farle duemila

anni di storia di Francia. (... $)^{110}$

Le due poesie scritte a distanza di un decennio una dall'altra hanno in comune soltanto la dedica e il genere letterario, per il resto sono affatto diverse. Mentre nel primo madrigale la graziosa duchessa è per cosí dire affrancata degli attributi aristocratici e il poeta ne astrae la posizione sociale, la storia per far emergere il fattore natura, nel secondo il procedimento è proprio opposto: la natura vi è subordinata alla storia.

Quale posto occupino ad un tratto i temi di carattere socio-politico nelle considerazioni di Saba è illustrato fra l'altro anche da Scorciatoie e rac-

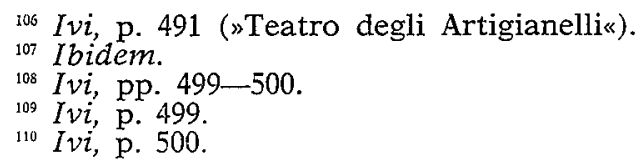


continit11 nonché dagli articoli che Saba scrisse nel dopoguerra. ${ }^{112}$ La serie di aforismi pubblicati nella prima metà del 1945 nella rivista La Nuova Europa di Salvatorelli si chiude con questa considerazione: "Dall'alto del suo altoparlante il dott. Goebbels attossisca il mondo. Nel limite delle mie forze, cerco, dalle colonne della, Nuova Europa', di disintossicarlo con ,Scorciatoie' (...)«. ${ }^{113}$ Tanto è l'engagement che avoca per sé l'estensore di quelle note.

Scorciatoie d'altro canto discoprono anche i limiti dell'impegno politico di Saba. Se guardiamo un po' da vicino i contributi che il nostro si propone di dare alla causa comune, vediamo che di impegno politico si può parlare soltanto nella misura in cui il poeta affronta temi di per se politici, mentre nel trattarli il piú delle volte astrae l'aspetto sociologico per tutto ridurre a un problema di psicologia individuale. I temi sociali sono affrontati partendo dalla premessa che mnon è, alla base, una questione economica ma psicologica. Solo secondarianente (perchè l'uomo è quello che è) diventa di spettanza degli economisti .11 Il concetto chiave con cui opera il Saba di Scorciatoie è »l'inconscio ${ }^{115}$ La visuale da cui affronta tutti i problemi sono di stretta osservanza freudiana e da questa prospettiva il poeta guarda agli avvenimenti politici, ai fatti letterari, interpreta la storia e racconta aneddoti. ${ }^{116}$ Alla realtà Saba non guarda neppure ora da posizioni che lo impegnerebbero davvero, il suo resta tuttora »il partito dei psicanalisti«. Il male contro il quele lotta è un male relegato nella sfera dell'inconscio e Hitler stesso in sostanza altro non è che "inconscio tenebroso, $^{117}$ l'esorcismo psicanalitico infine unico antidoto contro il male che affligge l'umanità.

L'engagement proprio del Saba subito dopo la liberazione risulta peraltro fenomeno effimero. Ben presto il discorso del poeta si attesta su

${ }^{111}$ U. Saba, Scorciatoie e raccontini, Milano, Mondadori, 1946.

112 Particolarmente interessanti si presentano gli articoli "Inferno e paradiso di Trieste" (Prose, pp. 817-822) e "Se fossi nominato governatore di Trieste" (Prose, pp. 823-826) dove Saba affronta la questione della convivenza fra gli italiani e gli sloveni a Trieste; si tratta - come l'autore stesso commenta - di un'»adeguazione ai tristi tempi di due versi giovanili scritti nel lontano 1912 (...) che (...) dicevano

E tu concili l'italo e lo slavo,

a tarda notte, lungo il tuo bigliardo.«

(Prose, pp. 823-824).

${ }^{113}$ U. Saba, Prose, op. cit., p. 294.

${ }^{114} I v i$, p. 269. Le sottolineature sono di Saba, secondo il quale ciò che contraddistingue gli »uomini politici« e rende efficace la loro azione è in primo luogo la volontà di potenza (Prose, p. 316).

${ }^{115}$ Le interpretazioni sabiane si richiamano qui sempre al »linguaggio dell'inconscio (Prose, p. 306).

${ }^{116}$ Il punto di vista cosí definito permette al nostro deduzioni ed accostamenti che, anche se non sempre convincono, meritano la massima attenzione in quanto rivelatori dell'aderenza alle premesse psicanalitiche che l'autore ha fatto proprie. Si notino per esempio le conclusioni che Saba deduce dal fatto che "Gli italiani sono l'unico popolo (...) che abbiano a base della loro storia (o della loro leggenda) un fratricidio « (Prose, p. 260), o l'analogia che intercorrerebbe fra »il cancro e il fascismo« quali fenomeni tipici del Novecento (Prose, pp. 278-279). Non meno interessanti si presentano le interpretazioni che Saba dà della Laura di Petrarca (Prose, pp. 264-265) o di un sonetto di Foscolo (Ivi, p. 325) imponendosi come promotore della critica psicanalitica in Italia.

${ }_{117}$ U. Saba, Prose, op. cit., p. 321. 
posizioni affatto apolitiche. In Scorciatoie si sfoga in forma estroversa il raptus psicanalitico che avevamo notato ai suoi esordi poetici. E il vecchio motivo ricaccia in secondo piano tutti gli altri impulsi.

Sfogliando le raccolte scritte nel dopoguerra, quelle cioè che comprendono le ultime liriche sabiane, si ha l'impressione che il Canzoniere nella parte finale non sia per niente meno vario che all'inizio, e se si avvertono certe differenze fra i singoli cicli ne sono nondimeno individuabili anche i denominatori comuni. Con sorprendente coerenza e tenacia il poeta rimane fedele alle già note direttrici tematiche e persino ai vecchi motivi.

Un bell'esempio di raffigurazione in cui riconosciamo subito Saba è dato dalla strofa centrale della già citata poesia Teatro degli artigianelli quando dice:

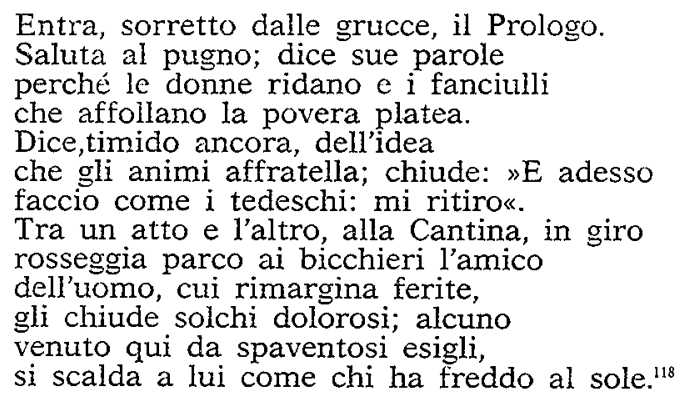

Di analoghe descrizioni sono fatti i "raccontini« quali L'osteria romana ${ }^{119}$ che richiamano alla memoria del lettore la vecchia parola d'ordine sabiana »'immettere la mia dentro la calda / vita di tutti«120 ovvero il motivo da cui il motto origina.

Dal punto di vista tematico interessante anche Opicina 1947,121 ultima poesia politica di Saba, pubblicata nella raccolta postuma Epigrafe. La scena che vi viene proposta è un ambiente sloveno con forte coscienza nazionale che fa sentire al poeta e agli altri parlanti italiano che sono degli estranei. Il poeta è conscio del proprio isolamento:

\footnotetext{
Due vecchie ebree, testarde villeggianti,

io, quel ragazzo, parlavamo ancora

lassú italiano, tra i sassi e l'abete. ${ }^{122}$
}

Il gruppo che in un ambiente straniero vuole testardamente rappresentare l'italianità non è senza ragione indicato come ebreo. L'immagine della terzina adombra il ruolo che a favore dell'italianità in una situazione tanto esposta ebbero gli ebrei senza per questo averne rinconoscimento da parte italiana. $\mathrm{E} i$ versi che chiudono il sonetto sono un rimprovero all'ingrata Italia che cosí com'è tanta fedeltà non si merita:

${ }^{118}$ U. Saba, Il Canzoniere (1900-1954), op. cit. 491.

119 U. Saba, Prose, op. cit., 196. Si vedano anche i »raccontini«: "Celsa« (Ivi, p. 356), »Italia mia« (Ivi, pp. 362-363), »Adesso che la guerra è finita (Ivi, pp. 364-365) e altri del genere.

"20 U. Saba, Il Canzoniere (1900-1954), p. 312 ("Il borgo«).

"121 Ivi, p. 612.

122 Ibidem. 
Dopo il nero fascista il nero prete;

questa è l'Italia, e lo sai. Perché allora -

diceva il mio compagno - aver rimpianti? ${ }^{223}$

Il motivo dell'estraneità che conosciamo fin dai primi componimenti, nelle ultime raccolte del Canzoniere si esplicita in vario modo. Una volta passati gli »spaventosi esigli« caratteristici specie delle poesie di Ultime cose il tema dell'esclusione assume una diversa motivazione mentre restano identici i moduli dell'impostazione tematica. All'esperienza che è alla base del lamento del "proscritto politico si sostituisce il pensiero della vecchiaia pur conservandosi immutata la metaforica: anche il senso della senescenza nel linguaggio sabiano si configura quale senso dell'estraniazione. Di questo il poeta è cosciente, come si vede dalla lettera in cui scrive all'amico: „Vedi tutti i rapporti tra me e la vecchiaia e tutte le esclusioni che ho provato un sollievo a buttare su di lei«. ${ }^{124}$

Già in 1944 la parola chiave diventa "sopravissuto ",25 già allora il poeta si rassegna al pensiero che il tempo in cui vive non è piú suo, non gli appartiene. Il »disoccupato ${ }^{126}$ con cui il poeta si identifica »Cantò coi soldati d'un altra / guerra«. ${ }^{127}$ Prima ancora che vengano dimenticate le "spaventose vicende ${ }^{128}$ il poeta è assalito da un angoscioso assillo:

\section{(...) Che ci faccio adesso}

che sono vecchio, che tutto s'innova, che il passato è macerie $(. .)^{129}$

Da qui lo sfogo con cui chiude il Canzoniere del 1945 "Ho scritto fine al mio lavoro ${ }^{130}$ col ritornello $\mathrm{Si}$ fa notte ${ }^{131}$ e altre espressioni di congedo dalla poesia.

"Col buio alle porte «132 è un'altra espressione che contrassegna anche Mediterranee le quali peraltro testimoniano di una vena poetica tutt'altro che esangue. Piú che »ebbri canti ${ }^{133}$ ed effusioni erotiche richiamano la nostra attenzione anche qui metafore che esprimono un senso di esclusione. Sintomatico è comunque già il fatto che tra Mediterranee si siano potute inserire anche poesie del 1940, 1941, 1942 allorché »superstite allegria «134 poteva indicare soltanto un accidente capace di dare ancor maggior rilievo alla generale tristezza.

${ }^{123}$ Ibidem. Nell'opera di Saba potrebbero essere indicate altre manifestazioni del risentimento qui espresso in maniera cosí esplicita.

${ }^{124}$ Cit. dalla lettera che Saba scrisse a Debenedetti il 3 settembre 1946 (V. Lettere di Saba, »Nuovi argomenti«, novembre-dicembre 1959, p. 27.

${ }^{125}$ Dalla lirica Vecchio camino che si conclude con il verso: "Vecchio sei come me, sopravvissuto. - Il Canzoniere (1900-1954), p. 493.

126 »Disoccupato«, Il Canzoniere (1900-1954), p. 492.

127 Ibidem.

${ }^{128}$ V. la seconda strofa della già citata poesia »Avevo", Il Canzoniere (19001954, p. 489.

129 Ibidern.

${ }^{130}$ La visita, poesia dedicata a Bruno e Maria Sanguinetti, Il Canzoniere (1900 fino a 1954), p. 502.

${ }^{131}$ Ibidem.

132 „Ebbri canti«, Il Canzoniere (1900-1954), p. 521.

${ }^{133}$ Ibidem.

134 "Dal vero«, Il Canzoniere $(1900-1954)$, p. 513. 
Nelle poesie scritte nel 1946 emerge un altro stato d'animo, pur tuttavia gli esiti delle autodefinizioni sabiane perciò non cambiano: i lamenti ispirati dagli »orrori del tempo ${ }^{135}$ seguono gli stessi moduli espressivi delle

(...) poesie che sono

ultime voci d'uno sulla terra, ${ }^{136}$

con sempre maggior insistenza si fa avanti l'immagine del

(..) sentirmi inerme
escluso $(. \ldots \ldots \ldots)^{137}$

Il leitmotiv di Mediterranee è suggerito già nel prologo della raccolta; e con maggior evidenza ancora emerge nell'epilogo dove leggiamo:

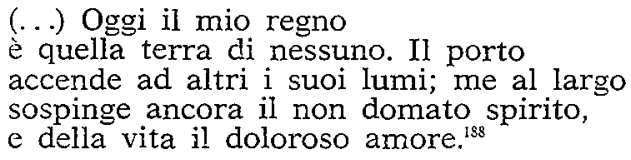

Le ben note costanti tematiche facilmente si riconoscono anche nelle raccolte Uccelli, Quasi un racconto e Sei poesie della vecchiaia con cui si chiude l'opera sabiana. In quali condizioni psichiche nascano le raccolte lo dicono già i prologhi che accompagnano la pubblicazione, le obbligatorie prefazioni nelle quali il poeta lamenta che »è dalla metà circa del 1947 che ho cominciato a sentirmi morire alle cose «139 esprimendo la convinzione »di non essere oramai che un peso morto sulla superficie della terra, di non avere nulla da fare o da dire in un mondo che non è piú miou. ${ }^{140}$ Il settantenne poeta si meraviglia già »del nudo fatto di aver potuto scrivere le poesie «.14i Nella prefazione a Quasi un racconto Saba esprime persino un "senso di rimorso e quasi di vergogna "142 di scrivere ancora. Analoghe considerazioni si ripetono anche nei versi; infatti la chiusa della raccolta citata dice appunto:

Questo libro che a te dava conforto,

buon lettore, è vergogna a chi lo crebbe.

Parlava come un vivo ed era (avrebbe

dovuto, per decenza, essere) morto. ${ }^{143}$

Tutte le poesie di Saba del periodo che la storia della letteratura indica come »la sua Quinta Stagione «144 a prima vista non sembrano concordare con tali effusioni. Uccelli, che costituiscono il motivo centrale del periodo in questione, si mostrano nel ruolo di esaltazione del ,bios', in un ruolo

135 »Libreria antiquaria«, Il Canzoniere (1900-1954), 558.

136 "Lettera«, Il Canzoniere (1900-1954), p. 613.

137 Dalla già citata "Libreria antiquaria».

136 "Ulisse", Il Canzoniere (1900-1954), p. 533.

${ }_{139}$ Prefazione ad »Uccelli«, Il Canzoniere (1900-1954), p. 537.

${ }^{140}$ Ivi, p. 538.

${ }^{1+1}$ Ivi, p. 537.

${ }_{142}$ Prefazione a "Quasi un racconto«, Il Canzoniere (1900-1954), p. 555.

${ }^{143}$ "Al lettore«, Il Canzoniere (1900-1954), p. 595.

${ }^{144}$ V. il saggio di Giacomo Debenedetti: La sua Quinta stagione (Intermezzo, op. cit., pp. $81-98$ ). 
dunque con cui il motivo che diciamo trovò rilievo già nei versi scritti più di quarant'anni prima. Gli attributi con cui viene contrassegnato il prototipo di Uccelli sono già nel prologo: "ingordo libero feroce «; ${ }^{145}$ l'»impeto gioioso « ${ }^{146}$ del Pettirosso che "si sgola ${ }^{147}$ a inizio di raccolta rappresenta un'immagine peculiare quant'altre mai. La poesia che ha dato il titolo a tutta la raccolta dice:

L'alata

genia che adoro - che n'è al mondo tanta! -

varia d'usi e costumi, ebbra di vita,

si sveglia e canta. ${ }^{\mathrm{I}+8}$

A conclusione il Rosignolo canta:

La dolcezza del mondo è una.

Solo a lei canto al lume della luna. ${ }^{149}$

L'esaltazione del bios' non è nel Canzoniere mai cosí rilevata come in Uccelli. Nella raccolta piú che in qualsiasi precedente trova espressione anche il presupposto che ha sempre accompagnato le rafigurazioni sabiane di una elementare gioia di vivere; il noto contrasto tra l'idea che il poeta ha della propria condizione e l'idealizzato atteggiamento esistenziale che gli si manifesta quale sarcana felicità di vivere« non è mai impostato cosí come nelle poesie del 1948: che sono un inno alla vita sciolto dalla posizione distaccata del vecchio che sa di aver fatto il proprio tempo e che, per cosí dire, guarda alla vita da fuori. Chi ignorasse questa premessa trascurerebbe un elemento essenziale del messaggio sabiano. Un'intrepretazione cosí si impone fin dal primo verso della raccolta. Il pensiero con cui iniziano Uccelli e che si stacca completamente isolato dal contesto dice:

Trattenerti, volessi anche, non posso. ${ }^{150}$

Verso che convalida la lettura di Debenedetti secondo cui Uccelli sarebbero »un discorso a un interlocutore poi cancellato ${ }^{151}$ e che ricollegando la nostre raccolta con la poesia Vecchio e giovane ${ }^{152}$ della precedente Epigrafe, pone in primo piano proprio l'idea dell'ineluttabilità della rinuncia all'amore e alla vita ovvero la conclusione: "Tu affretta, se puoi, tua morte ${ }^{153}$ In quel componimento gli attributi tutti sabiani di vita ed Eros andavano a »un ragazzo (...), bimbo - gatto in vista selvatico - (...) giovinetto tiranno , 154 nella seguente portatrice di vita diventa "l'alata genia«, ma il presupposto del discorso sabiano resta in sostanza immutato e il poeta lo rileva espressamente quando dice:

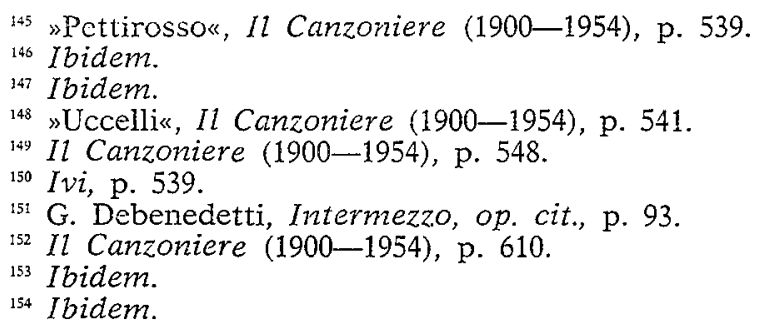


Qui tranquillo a riposo, dove penso

che ho dato invano, che la fine approssima,

piú mi piace quel cielo, quelle rondini,

quelle nubi. Non chiedo altro. ${ }^{155}$

La poesia di Saba è sempre stata autobiografica, perciò non stupisce il ruolo assegnato alla "vecchiaia« nelle ultime raccolte del Canzoniere. Il pensiero della vecchiaia non conduce però il poeta soltanto alle metafore dell'esclusione; a quest'idea si ricollega anche il caratteristico inno alla vita di Uccelli e Quasi un racconto. In questo congedo dalla vita si esprime infine la saggezza del nostro:

Mettere assieme i piú strani animali

$(. \ldots \ldots)$ e scrivere,

solo e con loro, qualche favoletta.

E questo il sogno della mia saggezza ultima. $(. \ldots \ldots \ldots)^{136}$

L'incentrarsi sui motivi presi dalla vita degli animali porta il poeta all'inevitabile ricupero dei già noti moduli del naturale. Né rappresentano una novità apostrofi come "Creature / di Dio e del sole«,, 157 o concetti come:

Uomo, la tua sventura è senza fondo.

Sei troppo e troppo poco. Con invidia

(tu pensi invece con disprezzo) guardi

gli animali, che immuni di riguardi

e di pudori, dicono la vita

a le sue leggi. (Ne dicono il fondo). ${ }^{158}$

Sono concetti che troviamo fin dalla prima parte del Canzoniere. E cosí pure abbiamo già avuto modo di conoscere i presupposti dello stato felice che Saba attribuisce agli esseri naturali, felice perché

$(\ldots \ldots \ldots)$ Non vede

sé, come vedo me stesso. Ed in questo

non vedersi è la sua felicità. ${ }^{\text {s9 }}$

Una tendenza anch'essa ormai nota è il suo "leggere «160 dal libro della creazione. Cosí i

(.........) Pianti

che vengono dal fondo della vita,

dall'esistere, e trovano la gola

sua d'uccelletta $(. \ldots \ldots \ldots)^{161}$

ripetono quanto aveva già detto »quell'uguale belato« della poesia $L a$ capra $^{162}$ scritta piú di quarant'anni prima. Nemmeno le »affinità«163 che il

155 "Cielo", Il Canzoniere (1900-1954), p. 540.

156 "Sognou, Il Canzoniere (1900-1954), p. 579.

${ }_{157}$ "Risveglio«, Il Canzoniere (1900-1954), p. 567.

iss »L'uomo e gli animali«, Il Canzoniere (1900-1954), p. 601.

1.59 »Palla d'oro«, Il Canzoniere (1900-1954), p. 561.

160 "Quasi una moralità", Il Canzoniere (1900-1954), p. 564.

${ }_{161}$ "Lina e la canarina azzurra», Il Canzoniere (1900-1954), p. 578.

${ }_{162}$ Il Canzoniere (1900-1954), p. 68.

${ }^{163}$ »Somiglianza«, Il Canzoniere (1900-1954), p. 565. 
poeta scopre fra animali e uomini compaiono per la prima volta in Quasi un racconto; si pensi a Mia moglie 164 che è dello stesso anno de La capra.

Nella raccolta Quasi un racconto dove il discorso sabiano si riduce alla cronaca della coesistenza coi modelli tali tendenze caratteristiche si riducono persino a maniera. Eloquente peraltro anche gli stereotipi che andrà ripetendo con l'affievolirsi dell'estro creativo: assieme a questi emergono anche tutte le piú incisive tendenze del discorso poetico per quanto in forme che qua e là suonano quasi grottesche, caricature delle soluzioni espressive di un tempo.

Esempi tipici di tale ripetitività troviamo specie fra le ultime cose di Saba raccolte sotto il comune titolo di Sei poesie della vecchiaia. Le immagini con cui il poeta esprime il senso di »esclusione " non vengono dalla constatazione che è "vecchio (...) sopravissuto ma del presupposto che anche in uno stato cui è costretto dalla conditio humana è menomato della parte che è di »tutti«:

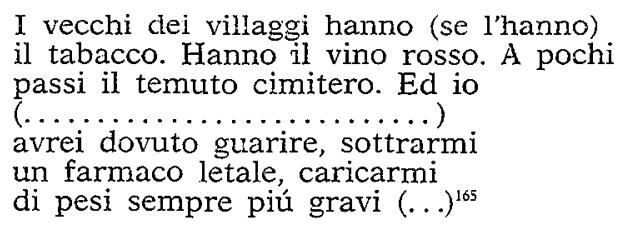

La variazione piú curiosa del vecchio motivo della diversità è data dal pensiero che:

V'ha chi solo si pensa ed indifeso.

Pensa che la sua carne ha un buon sapore.

Meglio - pensa - chi è in vista al cacciatore

passero che pernice..$^{166}$

Non meno bizzarra è Ultima dove leggiamo:

Guardo, donna, il tuo cane che adorato

ti adora. Ed io ... se penso alla mia vita!

$($ (...........................

Mai appartenni a qualcosa o a qualcuno.

$(\ldots \ldots \ldots \ldots \ldots \ldots \ldots \ldots \ldots \ldots)$

fui sempre un povero cane randagio. ${ }^{16} 7$

I versi con cui si chiude l'opera di Saba non sono proprio poetici, nondimeno esprimono quanto mai eloquentimente la linea tematica che può essere seguita fin dalle prime confessioni del poeta triestino.

${ }^{164}$ Il Canzoniere (1900-1954), pp. 64-66.

165 "I vecchi«, Il Canzoniere (1900-1954), p. 604.

${ }_{166}$ "Il poeta e il conformistau, Il Canzoniere (1900-1954), p. 603.

${ }^{167}$ Il Canzoniere (1900-1954), p. 606. 